\title{
AKT1 internal tandem duplications and point mutations are the genetic hallmarks of sclerosing pneumocytoma
}

\author{
Yi-Chen Yeh $\mathbb{D}^{1,2,3} \cdot$ Hsiang-Ling $\mathrm{Ho}^{1,4} \cdot$ Yu-Chung $\mathrm{Wu}^{3,5} \cdot$ Chin-Chen Pan ${ }^{1,3} \cdot$ Yu-Chao Wang $\mathbb{1}^{2,6} \cdot$ Teh-Ying Chou ${ }^{1,7}$
}

Received: 22 June 2019 / Revised: 5 August 2019 / Accepted: 6 August 2019 / Published online: 16 September 2019

(c) United States \& Canadian Academy of Pathology 2019

\begin{abstract}
Sclerosing pneumocytoma is a unique benign neoplasm of the lungs. The molecular alterations in sclerosing pneumocytoma are not well understood. In a previous whole-exome sequencing study, recurrent $A K T 1$ point mutation was observed in about half of the cases of sclerosing pneumocytoma. However, in the remaining half, cancer-related mutations have still not been identified. In this study, we first analyzed the raw sequence data from the previous whole-exome sequencing study (PRJNA297066 cohort). Using Genomon-ITDetector, a special software for detection of internal tandem duplications, we identified recurrent internal tandem duplications in the $A K T 1$ gene in 22 of the 44 tumor samples (50\%). All the cases positive for $A K T 1$ internal tandem duplications lacked $A K T 1$ point mutations. Next, we performed targeted next-generation sequencing in an independent cohort of sclerosing pneumocytoma from our hospital (VGH-TPE cohort), and again identified recurrent $A K T 1$ internal tandem duplications in 20 of the $40(50 \%)$ tumor samples analyzed. The internal tandem duplications resulted in duplications of 7 to 16 amino acids in a narrow region of the Pleckstrin homology domain of the AKT1 protein. This region contains the interaction interface between the Pleckstrin homology and kinase domains, which is known to play a critical role in the activation of the $A K T 1$ protein. Moreover, we found that $A K T 1$ internal tandem duplications were mutually exclusive of other forms of $A K T 1$ mutations, including point mutations and short indels. Taking all forms of $A K T 1$ mutations together, we detected $A K T 1$ mutations in almost all the sclerosing pneumocytomas in our study (PRJNA297066 cohort: 41 out of 44 cases, 93\%; VGH-TPE cohort: 40 out of 40 cases, 100\%). Our results suggest that AKT1 mutation is the genetic hallmark of sclerosing pneumocytoma. These results would help in better understanding of the pathogenesis of sclerosing pneumocytoma.
\end{abstract}

\section{Introduction}

Sclerosing pneumocytoma is an uncommon, but unique, neoplasm of the lungs. It occurs more frequently in middle-

Supplementary information The online version of this article (https:// doi.org/10.1038/s41379-019-0357-y) contains supplementary material, which is available to authorized users.

Yu-Chao Wang

yuchao@ym.edu.tw

$\triangle$ Teh-Ying Chou

tychou@vghtpe.gov.tw

1 Department of Pathology and Laboratory Medicine, Taipei Veterans General Hospital, Taipei, Taiwan

2 Institute of Biomedical Informatics, National Yang-Ming University, Taipei, Taiwan

3 School of Medicine, National Yang-Ming University, aged adults, and is predominant in females [1]. Although sclerosing pneumocytoma is generally considered a benign neoplasm, rare cases with regional lymph node metastases and local recurrence have been reported [2-4]. Diagnosis of sclerosing pneumocytoma can be challenging, especially on fine-needle aspiration and intraoperative frozen sections, and it is frequently misdiagnosed as lung adenocarcinoma $[5,6]$.

Taipei, Taiwan

4 Department of Biotechnology and Laboratory Science in Medicine, National Yang-Ming University, Taipei, Taiwan

5 Division of Thoracic Surgery, Department of Surgery, Taipei Veterans General Hospital, Taipei, Taiwan

6 Preventive Medicine Research Center, National Yang-Ming University, Taipei, Taiwan

7 Institute of Clinical Medicine, National Yang-Ming University, Taipei, Taiwan 
Sclerosing pneumocytoma has characteristic histopathological features. It usually displays a mixed solid, papillary, hemorrhagic, and/or sclerotic pattern, and contains two distinct cellular components, namely the round cells and the surface cells. Studies using laser microdissection and clonal analysis demonstrated that the round cells and the surface cells exhibit a uniform pattern of monoclonality, indicating that both the cell types likely originate from a common precursor [7, 8].

The histogenesis and pathogenesis of sclerosing pneumocytoma remain largely unknown at present. The molecular alterations in sclerosing pneumocytoma have been investigated in many studies. It has been shown that sclerosing pneumocytoma frequently harbors nonspecific microsatellite alterations in $\mathrm{p} 16$ and $\mathrm{Rb}$ tumor suppressor genes, but lacks alterations involving EGFR, HER-2, and $K$ $R A S$ [9]. Besides, nuclear expression of $\beta$-catenin proteins is frequently seen in sclerosing pneumocytoma, indicating that $\mathrm{Wnt} / \beta$-catenin pathway is involved in the pathogenesis. However, mutation in the exon 3 of $\beta$-catenin gene is rarely found in this tumor [10].

Recently, Jung et al. conducted a large-scale study using whole-exome sequencing to investigate genomic alterations in sclerosing pneumocytoma. They identified a high frequency of $A K T 1$ point mutations (overall 31 of $68,46 \%$ ) and recurrent $\beta$-catenin mutations (overall 3 of $68,4 \%$ ) [11]. This provides a milestone in understanding the molecular pathogenesis of sclerosing pneumocytoma. However, around half of the cases lacked identifiable cancer-related mutations in their study. This result has been particularly intriguing to us. Because sclerosing pneumocytoma is a disease with distinct and relatively uniform histopathological features, we expected that there should be a common genetic alteration in the majority of cases. We, therefore, speculated that sclerosing pneumocytoma might harbor certain kinds of genetic changes, which are difficult to detect by standard whole-exome sequencing analysis, such as structural variations, large insertions/deletions, and alterations in repetitive DNA sequences or non-coding genomic regions [12]. Internal tandem duplication, which may involve duplication of several tens to several hundreds of nucleotides within the coding exons, is also a probable genetic alteration. Internal tandem duplication is notoriously difficult to detect by standard bioinformatics pipelines, and specialized bioinformatics tools are usually required to identify this unique genetic change [13-15].

In the present study, we investigated the molecular alterations in sclerosing pneumocytoma, with a primary focus on internal tandem duplication. Using GenomonITDetector, a software package designed specifically for internal tandem duplication detection from cancer genome sequencing data [15], we discovered recurrent internal tandem duplications in AKTl gene in half of the cases.
Moreover, we found that $A K T 1$ internal tandem duplications were mutually exclusive of other forms of $A K T 1$ mutations, including point mutations and short indels. Taking all forms of $A K T 1$ mutations together, we were able to detect $A K T 1$ mutations in nearly all the sclerosing pneumocytoma cases in our study. These results suggest that AKTl mutations are the major molecular alterations in sclerosing pneumocytoma.

\section{Materials and methods}

\section{Analysis of PRJNA297066 sclerosing pneumocytoma cohort data}

First, we analyzed the publicly available next-generation sequencing dataset for sclerosing pneumocytoma using bioinformatics tools for detecting internal tandem duplication to discover potentially unidentified molecular changes. Briefly, the raw sequence data from a total of 44 paired normal and sclerosing pneumocytoma tumor samples in the previous whole-exome sequencing study by Jung et al. (Project ID: PRJNA297066) [11] were download from the Sequence Read Archive database (https://www.ncbi.nlm.nih. gov/sra). The downloaded SRA files were converted to fastq files by fastq-dump in the NCBI SRA Toolkit. The sequencing reads were then aligned to the human reference genome (UCSC hg19) by Burrows-Wheeler Alignment tool to generate the aligned BAM files [16]. Genomon-ITDetector, a software package for detection of internal tandem duplications in cancer genome sequencing data, was then used to detect internal tandem duplications in the aligned BAM files [15]. Manual reviews and confirmation of the internal tandem duplications were done with Integrative Genomics Viewer [17]. The data of $A K T 1$ and $\beta$-catenin point mutations were retrieved from the manuscript by Jung et al. [11].

\section{VGH-TPE sclerosing pneumocytoma cohort samples}

We also included an independent cohort of patients with sclerosing pneumocytoma who underwent surgical resection in Taipei Veterans General Hospital between 2007 and 2017 (VGH-TPE cohort). The samples from this cohort consisted of 40 sclerosing pneumocytoma tumor samples, 25 of which had paired normal lung samples. All the tissues had been fixed in formalin before being embedded in paraffin blocks. The hematoxylin and eosin-stained slides were reviewed by a pathologist (Y.C.Y). The percentages of histological growth patterns, including hemorrhagic, solid, papillary, and sclerotic, were semi-quantitatively recorded with $5 \%$ increments for each tumor. The predominant histological pattern was then defined as the pattern with the highest percentage. 


\section{Targeted next-generation sequencing in VGH-TPE sclerosing pneumocytoma cohort}

We performed targeted next-generation sequencing to sequence all exons of $A K T 1$ and $\beta$-catenin genes in the tumor and paired normal samples in the VGH-TPE sclerosing pneumocytoma cohort. Briefly, sections cut from formalinfixed paraffin-embedded specimens were used for DNA extraction. The collected DNA was purified with GeneRead DNA FFPE Kit (Qiagen, Inc.), according to the manufacturer's instructions. The quality and quantity of the DNA were verified using NanoDrop 2000 (Thermo Fisher Scientific, Inc.) and Fragment Analyzer (Agilent formerly Advanced Analytical (AATI), Inc.). A customized DNA panel was designed using the online tool, QIAseq Targeted DNA Custom Panel Builder, covering all coding regions of $A K T 1$ and $\beta$-catenin genes. Genomic DNA samples (100 ng) were used for library preparation with multiplex PCR target enrichment panel, according to the manufacturer's instructions. The purified libraries were pooled and then sequenced with MiSeq instrument (Illumina, Inc.).

For single nucleotide variations and short indels, variant detection was performed by smCounter 2 workflow, a software package for detecting somatic variants in QIAseq Targeted DNA Panel [18]. Variant allele frequency was obtained from the variant unique molecular identifier allele fraction data in the reports generated by the smCounter2. Low-frequency variants (with variant allele frequency $<5 \%$ ) were filtered out because they are likely to represent subclonal mutations. Synonymous mutations were also filtered out.

For detection of internal tandem duplications, the process was similar to that used for PRJNA297066 sclerosing pneumocytoma cohort data. The reads were aligned with the hg19 human reference genome using the Burrows-Wheeler Alignment tool. Detection of internal tandem duplications was performed by Genomon-ITDetector. Manual reviews of the detected internal tandem duplications were conducted using the Integrative Genomics Viewer.

\section{Targeted PCR, gel electrophoresis, and Sanger sequencing}

Hematoxylin-eosin stained tissue slides were reviewed by the pathologist to select the tumor regions. The selected tumor region was dissected manually from the corresponding areas in consecutive tissue sections and, after deparaffinization, subjected to genomic DNA extraction. Briefly, the dissected tumor tissue was transferred to an eppendorf tube containing proteinase $\mathrm{K}$ solution. The tube was then incubated at $56^{\circ} \mathrm{C}$ for $16 \mathrm{~h}$, followed by an inactivation step at $95^{\circ} \mathrm{C}$ for $10 \mathrm{~min}$. Exons 3 and 4 of the $A K T 1$ gene (NCBI reference sequence:
NM_001014431.1) were amplified by PCR in a total volume of $25 \mu \mathrm{L}$ containing $100 \mathrm{ng}$ of template DNA, $1 \mathrm{X}$ Taq Master Mix Red (Ampliqon III), and $0.5 \mu \mathrm{M}$ of sense and antisense primers. The primer sequences are listed in Supplementary Table 1 . The sense and antisense primers used for amplification of $A K T 1$ exon 3 were $A K T 1$-E3S1 and $A K T 1$-E3A1, and those for exon 4 were AKT-E4S1 and $A K T 1$-E4A1, respectively. The PCR was carried out for 35 cycles of $95^{\circ} \mathrm{C}$ for $40 \mathrm{~s}, 56^{\circ} \mathrm{C}$ for $40 \mathrm{~s}$, and $72^{\circ} \mathrm{C}$ for $40 \mathrm{~s}$, followed by $5 \mathrm{~min}$ extension at $72^{\circ} \mathrm{C}$. The amplicons were electrophoresed on $4 \%$ agarose gels. For $A K T 1$ exon $3 \mathrm{E} 17 \mathrm{~K}$ mutation analysis, the PCR products were subjected to Sanger sequencing with $A K T 1$-E3A2 primers. For $A K T 1$ exon 4 internal tandem duplication analysis, the PCR products, with two major bands identified on agarose gels, were extracted, purified, and then subjected to TA-cloning using $\mathrm{T} \& \mathrm{~A}^{\mathrm{TM}}$ Cloning Vector Kit (Yeastern Biotech Co. Ltd., Taiwan), according to manufacturer's instructions. After transformation, single colonies were picked and subjected to Sanger sequencing with M13 forward primers.

\section{Statistical analysis}

The association of clinical and pathological characteristics with molecular alterations was analyzed by Student's $t$-test (for continuous variables), or chi-square test/Fisher's exact test (for categorical variables), when appropriate. Analyses were performed using SPSS software (IBM, Armonk, NY, USA). A $P$-value $<0.05$ was considered to be statistically significant.

\section{Results}

\section{Discovery of $A K T 1$-internal tandem duplication in PRJNA297066 sclerosing pneumocytoma cohort}

We analyzed the whole-exome sequencing raw sequence data from PRJNA297066 sclerosing pneumocytoma cohort using the Genomon-ITDetector software, and then performed manual review with Integrative Genomics Viewer. Surprisingly, a high frequency of recurrent in-frame internal tandem duplications in the $A K T 1$ gene was discovered in 22 of the 44 tumor samples $(50 \%)$ (Table 1, Fig. 1 and Supplementary Fig. 1). The internal tandem duplications were clustered in exon 4 of $A K T 1$, which encodes the Pleckstrin homology domain of the AKT1 protein. The internal tandem duplications were predicted to result in duplications of 7 to 15 amino acids in this region (Fig. 2a). In a subset of the cases (SH54T, SH67T, SH57T, SH41T, SH58T, SH09T, and SH03T), a novel junctional amino acid was introduced by the internal tandem duplications. In 
Table 1 AKT1 internal tandem duplication and other mutations in AKT1 and $\beta$-catenin genes in the PRJNA297066 sclerosing pneumocytoma cohort

\begin{tabular}{|c|c|c|c|}
\hline Sample ID & Patient's sex & $A K T 1$ internal tandem duplication & $\begin{array}{l}\text { Other mutations in } A K T 1 \text { and } \\
\beta \text {-catenin genes }\end{array}$ \\
\hline SH01T & Female & & $A K T 1$ p.E17K \\
\hline $\mathrm{SH} 02 \mathrm{~T}$ & Male & p.R67_Q79dup & \\
\hline SH03T & Female & p.V83_I84insSCLQWSTV & \\
\hline SH04T & Female & & $A K T 1$ p.N53H, p.E49K \\
\hline SH05T & Female & p.K64_L78dup & \\
\hline SH06T-1 & Female & & $A K T 1$ p.F55Y, p.L52R \\
\hline SH06T-2 & Female & & $A K T 1$ p.E17K \\
\hline SH07T & Female & p.M63_R76dup & \\
\hline SH08T & Female & p.R67_Q79dup & \\
\hline SH09T & Female & p.R76_C77insW+p.P70_R76dup & \\
\hline SH10T & Female & p.P68_Q79dup & \\
\hline SH11T & Female & p.T65_C77dup & \\
\hline SH18T & Female & & $\beta$-catenin $\mathrm{p} . \mathrm{S} 37 \mathrm{~F}$ \\
\hline SH20T & Female & & $A K T 1$ p.E17K \\
\hline $\mathrm{SH} 23 \mathrm{~T}$ & Female & & AKT1 p.E17K \\
\hline $\mathrm{SH} 25 \mathrm{~T}$ & Female & & $A K T 1$ p.E17K \\
\hline $\mathrm{SH} 34 \mathrm{~T}$ & Female & & $A K T 1$ p.E17K \\
\hline SH36T & Female & & $A K T 1$ p.E17K \\
\hline SH37T & Male & & AKT1 p.Q79K, p.W80R \\
\hline SH40T & Female & & $A K T 1$ p.E17K \\
\hline SH41T & Female & p.Q79_W80insC+p.R67_Q79dup & \\
\hline SH42T & Female & & $A K T 1$ p.E17K, p.I19L \\
\hline SH43T & Female & & \\
\hline SH46T & Female & p.M63_R76dup & \\
\hline SH47T & Female & p.K64_L78dup & \\
\hline $\mathrm{SH} 48 \mathrm{~T}$ & Female & & $A K T 1$ p.E17K \\
\hline SH49T & Female & p.R67_L78dup & \\
\hline SH50T & Male & & \\
\hline SH51T & Female & p.R67_L78dup & \\
\hline SH52T & Female & & $A K T 1$ p.E17K \\
\hline SH53T & Male & p.R67_L78dup & \\
\hline SH54T & Female & p.C77_L78insP+p.E66_C77dup & \\
\hline SH55T & Female & & $A K T 1$ p.E17K \\
\hline SH56T & Female & & AKT1 p.Q79K, p.W80R \\
\hline SH57T & Female & p.C77_L78insQ+p.R67_C77dup & \\
\hline SH58T & Female & p.R76_C77insW+p.P68_R76dup & \\
\hline SH59T & Female & p.R67_C77dup & \\
\hline SH60T & Female & & AKT1 p.Q79K, p.W80R \\
\hline SH61T & Female & & $A K T 1$ p.Q79K, p.W80G \\
\hline SH62T & Female & & AKT1 p.Q79K, p.W80R \\
\hline SH63T & Female & p.R67_Q79dup & \\
\hline SH64T & Female & p.R67_L78dup & \\
\hline SH66T & Female & p.T65_C77dup & $\beta$-catenin $\mathrm{p} . \mathrm{S} 37 \mathrm{~F}$ \\
\hline SH67T & Female & p.R76_C77insS+p.E66_R76dup & \\
\hline
\end{tabular}




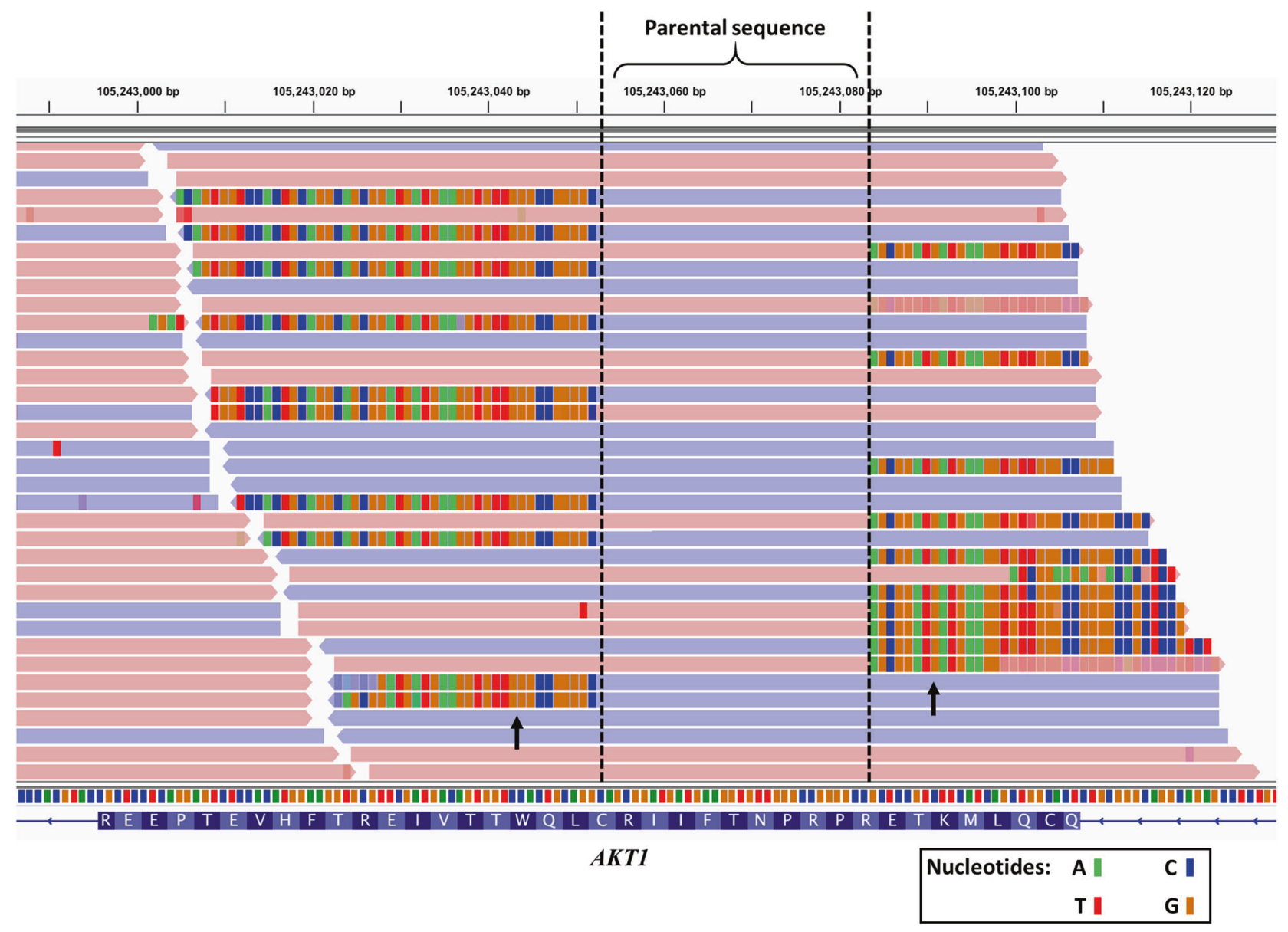

Fig. 1 Representative screenshot showing alignments around the $A K T 1$ exon 4 in a tumor sample (SH58T) positive for AKT1 internal tandem duplication, using the Integrative Genomics Viewer. The regions between the two dotted lines are the parental sequences of

duplicated nucleotides. The duplicated nucleotides (arrow) are aligned either to the right or left of the parental sequences, and they are marked as soft-clipped sequences by the alignment software. This is a characteristic feature of internal tandem duplications

SH03T, there was an additional point mutation in the middle of the duplicated segment, which resulted in a threonine (T) to serine (S) substitution. The $A K T 1$-internal tandem duplications were only detected in the tumor samples, and were absent in all the normal samples. Moreover, we found that $A K T 1$-internal tandem duplications and $A K T 1$ point mutations were mutually exclusive, and were found in 41 of the 44 tumor samples $(93 \%)$.

\section{Validation of $A K T 1$-internal tandem duplications in the VGH-TPE sclerosing pneumocytoma cohort}

To validate our findings in the PRJNA297066 sclerosing pneumocytoma cohort, we performed targeted nextgeneration sequencing to sequence all the exons of $A K T 1$ and $\beta$-catenin genes in an independent cohort (VGH-TPE cohort). This cohort consisted of 40 patients with sclerosing pneumocytoma who underwent surgery in our hospital. Similar to the findings in the PRJNA297066 cohort, we detected recurrent in-frame internal tandem duplications in the $A K T 1$ gene in 20 of the 40 tumor samples in the VGH-TPE cohort (50\%) (Table 2 and Supplementary Fig. 2). The internal tandem duplications clustered in exactly the same region of the $A K T 1$ protein Pleckstrin homology domain as in the PRJNA297066 cohort and were predicted to result in duplications of 7 to 16 amino acids in this region (Fig. 2b). In a subset of the cases (V18T, V20T, V28T, V16T, V35T, V37T, V33T, and V04T), a novel junctional amino acid was introduced by the internal tandem duplications, which was also very similar to that observed in the PRJNA297066 cohort. In addition, in the case V23T, there was a point mutation in the middle of the duplicated segment, which resulted in a proline (P) to alanine (A) substitution. This finding was similar to that obtained for the case SH03T in the PRJNA297066 cohort.

The analysis of $A K T 1$ exon 4 using genomic PCR and electrophoresis on a $4 \%$ agarose gel revealed that cases carrying AKTl-internal tandem duplication yielded a higher molecular weight product (Fig. 3a). Sanger 
a

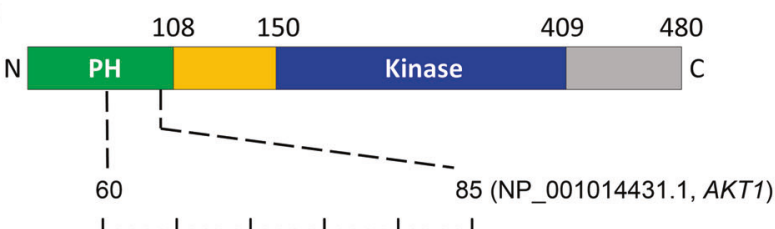

AKT1_wt CQIMKTERPRPNTFIIRCLQWTTVIE

SH46T CQLMKTERPRPNTFIIRMKTERPRPNTFIIRCLQWTTVIE SH07T CQLMKTERPRPNTEIIRMKTERPRPNTEIIRCLQWTTVIE SH05T CQLMKTERPRPNTFIIRCLKTERPRPNTFIIRCIQWTTVIE SH47T CQLMKTERPRPNTFIIRCLKTERPRPNTFIIRCLQWTTVIE SH11T CQLMKTERPRPNTFIIRCTERPRPNTFIIRCLQWTTVIE SH66T CQLMKTERPRPNTFIIRCTERPRPNTFIIRCLQWTTVIE SH54T CQLMKTERPRPNTFIIRCPERPRPNTFIIRCLQWTTVIE SH67T CQLMKTERPRPNTFIIRSERPRPNTEIIRCLQWTTVIE SH57T CQLMKTERPRPNTFIIRCQRPRPNTFIIRCLQWTTVIE SH59T CQLMKTERPRPNTFIIRCRPRPNTFIIRCLQWTTVIE SH51T CQLMKTERPRPNTEIIRCLRPRPNTFIIRCLQWTTVIE SH53T CQLMKTERPRPNTFIIRCLRPRPNTFIIRCLQWTTVIE SH64T CQLMKTERPRPNTFIIRCLRPRPNTFIIRCLQWTTVIE SH49T CQIMKTERPRPNTFIIRCLRPRPNTFIIRCLQWTTVIE SH63T CQLMKTERPRPNTFIIRCLORPRPNTFIIRCLQWTTVIE SH02T CQIMKTERPRPNTFIIRCLQRPRPNTFIIRCLQWTTVIE SH08T CQLMKTERPRPNTFIIRCLQRPRPNTFIIRCLQWTTVIE SH41T CQIMKTERPRPNTFIIRCLQCRPRPNTFIIRCLQWTTVIE SH58T CQLMKTERPRPNTFIIRWPRPNTFIIRCLQWTTVIE SH10T CQIMKTERPRPNTFIIRCIQPRPNTFIIRCLQWTTVIE SH09T CQLMKTERPRPNTFIIRWPNTFIIRCLQWTTVIE SH03T CQLMRTERPRPNTFIIRCLOWTTVSCLQWSTVIE

Fig. 2 a Schematic of the $A K T 1$ protein structure and predicted $A K T 1$ protein sequences from the tumor samples positive for internal tandem duplication in the PRJNA297066 cohort. The wild-type AKT1 protein sequence (amino acids 60-85) is shown on the top, with the predicted protein sequence of each internal tandem duplication-positive case shown below. The first segments of the duplicated sequences are shown in green, and the second segments are shown in blue. Novel junctional amino acids (red) were introduced by the internal tandem duplications in cases SH54T, SH67T, SH57T, SH41T, SH58T, SH09T, and SH03T. In SH03T, there was an additional point mutation in the middle of the duplicated segment, which resulted in a threonine (T) to serine (S) substitution. b Schematic of the AKT1 protein

sequencing of the distinct PCR products showed the presence of in-frame tandem duplications and the duplicated sequences were identical to those derived from targeted next-generation sequencing (Fig. 3b).

In addition to $A K T 1$-internal tandem duplication, we also detected other forms of AKT1 mutations in the sclerosing pneumocytoma tumor samples (Table 2), including p.E17K (six cases) (Fig. 4), p.E17K/p.E40K (one case), p.E17K/p.I19F (one case), p.E17K/p.W80G (one case), p.E49K/p.N53H (one case), p.Q79K/p.W80G (six cases), p.Q79K/p.W80R (two cases), pW80R/p. L78_Q79insKK (one case), and p.Q79_W80delinsR/p. T81delinsMPGA (one case). Most importantly, all these $A K T 1$ mutations were mutually exclusive with $A K T 1$ internal tandem duplications. Taking all forms of $A K T 1$ b

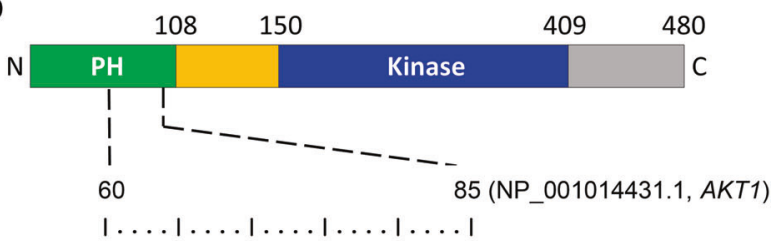

AKT1_Wt CQLMKTERPRPNTFIIRCLQWTTVIE

V26T CQLMKTERPRPNTEIIRQLMKTERPRPNTEIIRCLQWTTVIE

V10T CQLMKTERPRPNTFIIRMRTERPRPNTFIIRCLQWTTVIE

V15T CQLMRTERPRPNTFIIRCLRTERPRPNTFIIRCLQWTTVIE

V18T CQLMKTERPRPNTFIIRLKTERPRPNTFIIRCLQWTTVIE

V20T CQLMKTERPRPNTFIIRWKTERPRPNTEIIRCLQWTTVIE

V23T CQLMRTERPRPNTFIIRTERARPNTFIIRCLQWTTVIE

V28T CQLMKTERPRPNTFIIRCRTERPRPNTFIIRCLQWTTVIE

V29T CQLMKTERPRPNTFIIRCLQERPRPNTFIIRCLQWTTVIE

V14T CQLMRTERPRPNTEIIRCLQERPRPNTEIIRCLOWTTVIE

V16T CQLMKTERPRPNTFIIRCRERPRPNTFIIRCLQWTTVIE

V21T CQLMKTERPRPNTEIIRCLERPRPNTEIIRCLQWTTVIE

V24T CQIMKTERPRPNTFIIRCLORPRPNTFIIRCLOWTTVIE

V38T CQLMRTERPRPNTFIIRCLRPRPNTFIIRCLQWTTVIE

V35T CQLMKTERPRPNTFIIRWPRPNTFIIRCLQWTTVIE

V34T CQLMKTERPRPNTEIIRCLRPNTEIIRCLQWTTVIE

V37T CQLMRTERPRPNTFIIRWPNTFIIRCLQWTTVIE

V33T CQLMKTERPRPNTFIIRCLQWTNIRCLQWTTVIE

V31T CQLMKTERPRPNTFIIRCLOWTTVRCLOWTTVIE

V19T CQLMKTERPRPNTFIIRCLQWTTVRCLQWTTVIE

V04T CQLMKTERPRPNTFIIRCLQWTTVSCLQWTTVIE

structure and predicted $A K T 1$ protein sequences from the tumor samples positive for internal tandem duplications in the VGH-TPE cohort. The wild-type $A K T 1$ protein sequence (amino acids 60-85) is shown on the top, with the predicted protein sequence of each internal tandem duplication-positive case shown below. The first segments of the duplicated sequences are shown in green, and the second segments are shown in blue. Novel junctional amino acids (red) were introduced by the internal tandem duplications in cases V18T, V20T, V28T, V16T, V35T, V37T, V33T, and V04T. In V23T, there was an additional point mutation in the middle of the duplicated segment, which resulted in a proline (P) to alanine (A) substitution. $\mathrm{PH}$ Pleckstrin homology domain, Kinase Kinase domain N N-terminal, C C-terminal

mutations together (including point mutations, indels, and internal tandem duplications), all sclerosing pneumocytoma tumor samples in the VGH-TPE cohort contained one of the AKTl mutations.

With regard to $\beta$-catenin genes, we detected p.S45P point mutation in one single case (V09T). However, this case also harbored the $A K T 1$ p.E17K mutation. The variant allele frequency of $A K T 1$ p.E17K mutation was $36 \%$. In contrast, the variant allele frequency of $\beta$-catenin p.S45P mutation was only $9 \%$. The low variant allele frequency of $\beta$-catenin p.S45P mutation suggested that this mutation was present in a subclone of tumor cells, and was likely to be a secondary event during tumor progression.

No $A K T 1$ or $\beta$-catenin mutations were detected in normal samples of the VGH-TPE cohort. The pie charts of AKTI 
Table 2 AKT1 internal tandem duplications and other mutations in AKT1 and $\beta$-catenin genes in the VGH-TPE sclerosing pneumocytoma cohort

\begin{tabular}{|c|c|c|c|c|}
\hline Sample ID & Patient's sex & Histology & $A K T 1$ internal tandem duplication & $\begin{array}{l}\text { Other mutations in } A K T 1 \\
\text { and } \beta \text {-catenin genes }\end{array}$ \\
\hline V01T & Female & Papillary & & $A K T 1$ p.E17K \\
\hline V02T & Male & Hemorrhagic & & $A K T 1$ p.E17K; p.E40K \\
\hline V03T & Female & Hemorrhagic & & AKT1 p.Q79K; p.W80G \\
\hline V04T & Female & Papillary & p.V83_I84insS+p.C77_V83dup & \\
\hline V05T & Female & Solid & & $A K T 1$ p.E17K \\
\hline V06T & Female & Solid & & $A K T 1$ p.E17K; p.W80G \\
\hline V07T & Female & Hemorrhagic & & $A K T 1$ p.E17K \\
\hline V08T & Female & Papillary & & $A K T 1$ p.E17K; p.I19F \\
\hline V09T & Female & Hemorrhagic & & $\begin{array}{l}\text { AKT1 p.E17K; } \beta \text {-catenin } \mathrm{p} \text {. } \\
\text { S45P }\end{array}$ \\
\hline V10T & Female & Hemorrhagic & p.M63_R76dup & \\
\hline V11T & Male & Papillary & & $A K T 1$ p.Q79K; p.W80G \\
\hline V12T & Female & Solid & & $A K T 1$ p.Q79K; p.W80R \\
\hline V13T & Female & Solid & & $A K T 1$ p.Q79K; p.W80G \\
\hline V14T & Female & Hemorrhagic & p.E66_Q79dup & \\
\hline V15T & Female & Hemorrhagic & p.K64_L78dup & \\
\hline V16T & Female & Hemorrhagic & p.C77_L78insR+p.E66_C77dup & \\
\hline V17T & Female & Solid & & $A K T 1$ p.E17K \\
\hline V18T & Female & Sclerotic & p.R76_C77insL+p.K64_R76dup & \\
\hline V19T & Female & Hemorrhagic & p.R76_V83dup & \\
\hline V20T & Female & Papillary & $\begin{array}{l}\text { p.R76_C77insW+p. } \\
\text { K64_R76dup }\end{array}$ & \\
\hline V21T & Female & Solid & p.E66_L78dup & \\
\hline $\mathrm{V} 22 \mathrm{~T}$ & Male & Papillary & & $A K T 1$ p.Q79K; p.W80R \\
\hline V23T & Female & Papillary & p.I75_R76insKTERARPNTFII & \\
\hline $\mathrm{V} 24 \mathrm{~T}$ & Female & Papillary & p.R67_Q79dup & \\
\hline V25T & Male & Papillary & & $A K T 1$ p.Q79K; p.W80G \\
\hline V26T & Female & Papillary & p.Q61_R76dup & \\
\hline V27T & Female & Solid & & $A K T 1$ p.E49K; p.N53H \\
\hline V28T & Female & Papillary & p.C77_L78insR+p.T65_C77dup & \\
\hline V29T & Female & Solid & p.E66_Q79dup & \\
\hline V30T & Female & Solid & & $A K T 1$ p.E17K \\
\hline V31T & Female & Solid & p.R76_V83dup & \\
\hline V32T & Female & Sclerotic & & $\begin{array}{l}\text { AKT1 p.W80R; p. } \\
\text { L78_Q79insKK }\end{array}$ \\
\hline V33T & Female & Solid & pT81_T82insN+p.I75_T81dup & \\
\hline V34T & Female & Solid & p.R69_L78dup & \\
\hline V35T & Female & Solid & $\begin{array}{l}\text { p.R76_C77_insW+p. } \\
\text { P68_R76dup }\end{array}$ & \\
\hline V36T & Female & Papillary & & AKT1 p.Q79K; p.W80G \\
\hline V37T & Female & Hemorrhagic & p.R76_C77insW+p.P70_R76dup & \\
\hline V38T & Female & Papillary & p.R67_L78dup & \\
\hline V39T & Female & Hemorrhagic & & $\begin{array}{l}\text { AKT1 p.Q79_W80delinsR; } \\
\text { p.T81delinsMPGA }\end{array}$ \\
\hline V40T & Female & Hemorrhagic & & $A K T 1$ p.Q79K; p.W80G \\
\hline
\end{tabular}




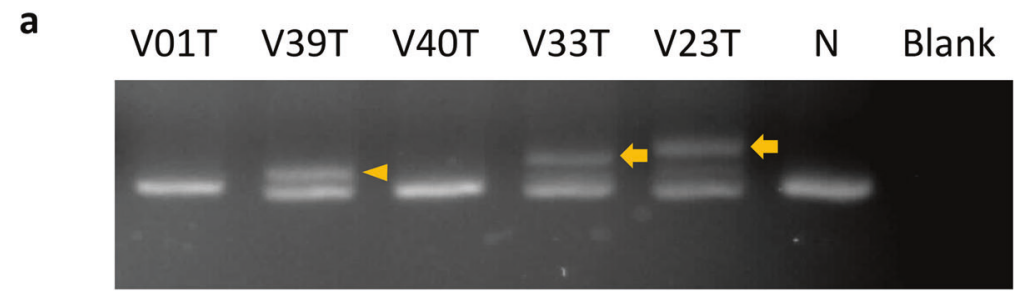

b
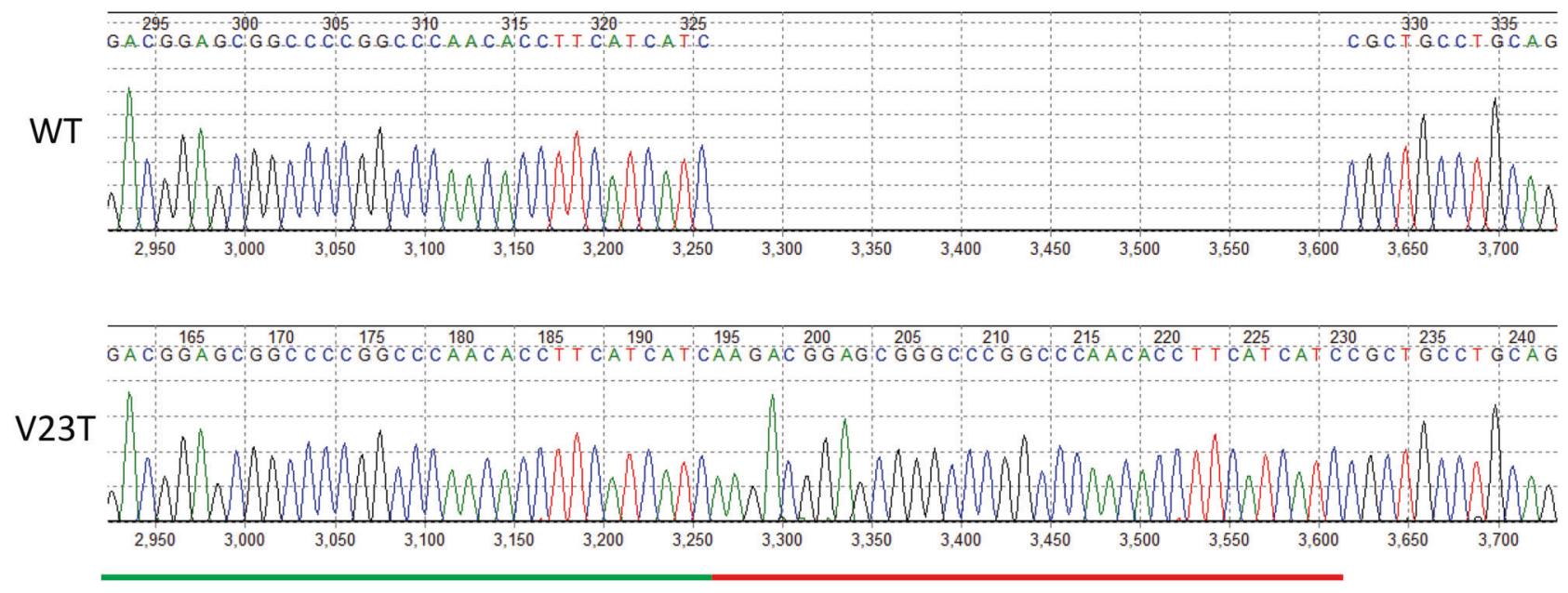

Parental

Fig. 3 a Targeted PCR and gel electrophoresis of $A K T 1$ exon 4 from five representative samples (V01T, V39T, V40T, V33T, and V23T) in the VGH-TPE cohort. In samples positive for $A K T 1$, internal tandem duplication (V33T and V23T), large PCR products corresponding to the internal tandem duplications were detected (arrow), in addition to the expected wild-type products. In samples V01T and V40T, which are negative for $A K T 1$ internal tandem duplication, only wild-type

and $\beta$-catenin mutations in the PRJNA297066 cohort and VGH-TPE cohort are shown in Fig. 5.

\section{Correlation between clinicopathological characteristics and AKT1 mutations in the VGH-TPE sclerosing pneumocytoma cohort}

We analyzed the correlation between clinicopathological characteristics and AKT1 mutations in the VGH-TPE cohort, and the results are summarized in Table 3. There were no significant differences in clinical characteristics or pathological features between patients with $A K T 1$-internal tandem duplication or other types of $A K T 1$ mutations (Fig. 6). We noticed that all the male patients in the VGHTPE cohort belonged to the group with other types of $A K T 1$ mutations. However, this was probably due to chance because we observed that two male patients in the PRJNA297066 cohort belonged to the group with AKT1internal tandem duplication (SH53T and SH02T).

\section{Duplication}

products were detected. In sample V39T, an additional PCR product, slightly larger than the wild-type product, was also detected (arrowhead), which corresponds to the complex AKT1 mutation of $\mathrm{p}$. Q79_W80delinsR and p.T81delinsMPGA in this case. b Sanger sequencing from a representative sample (V23T) positive for $A K T 1$ internal tandem duplication showed the duplicated sequence. $\mathrm{N}$ normal lung, WT wild type

\section{Discussion}

In this study, we demonstrate that $A K T 1$ mutation is present in nearly all the sclerosing pneumocytomas, with internal tandem duplication and point mutations being the most common forms. AKT1 belongs to the AKT kinase family, the members of which serve as critical downstream effectors of phosphatidylinositol-3 kinase (PI3K) signaling. Under basal conditions, AKT1 localizes to the cytosol, and maintains an inactive closed "PH-in" conformation through the interaction between its Pleckstrin homology and kinase domains [19]. Following activation of phosphatidylinositol-3 kinase by proliferative signals, the inactive $A K T 1$ in the cytosol is recruited to the plasma membrane through binding of the Pleckstrin homology domain. This results in a conformational change that separates the Pleckstrin homology and kinase domains, shifting AKTl to an open "PH-out" state. The PH-out state enables phosphorylation of Thr308 and Ser473 


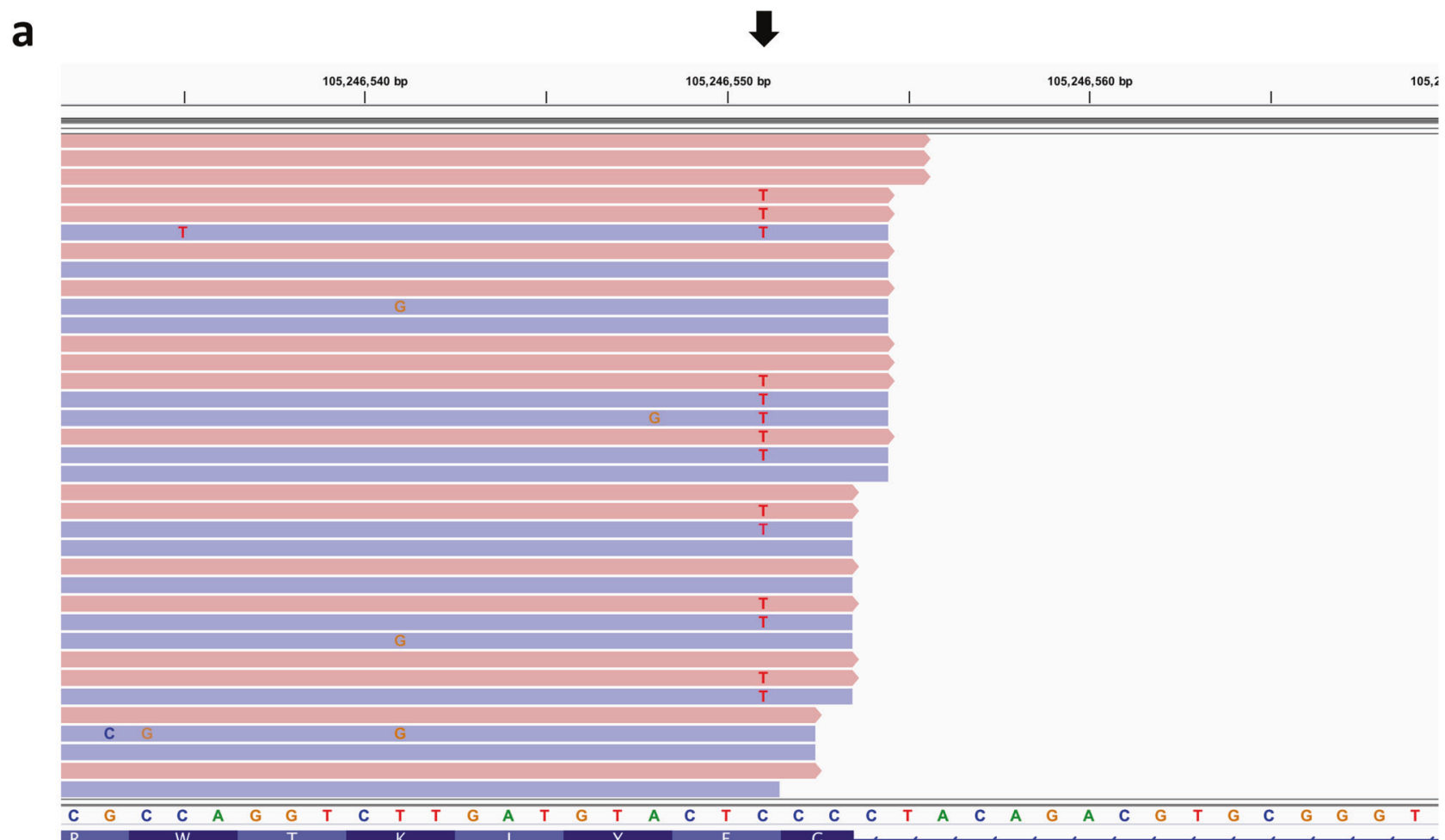

b

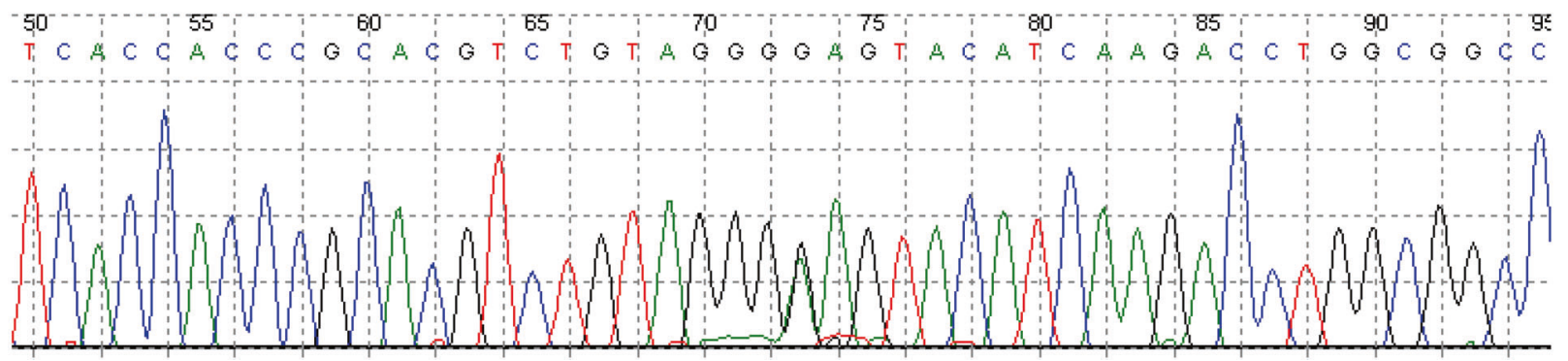

Fig. 4 a Screenshot for a representative sample (V01T) showing AKT1 p.E17K (c.49G>A) mutation, using Integrative Genomics Viewer. The reference sequence shown in Integrative Genomics Viewer is the antisense strand of $A K T 1$, and the corresponding nucleotide change for
p.E17K mutation is C>T mutation (arrow). b Sanger sequencing from the same sample confirmed the $A K T 1$ p.E17K (c.49G $>$ A) mutation (arrow)

In contrast, little is known regarding the role of $A K T 1$ internal tandem duplications in oncogenesis. In the literature, there are very few reports of $A K T 1$ internal tandem duplications in human cancers. To the best of our knowledge, only juvenile granulosa cell tumor and breast cancer have been reported to harbor $A K T 1$ internal tandem duplications $[23,24]$. The affected genomic region in juvenile granulosa cell tumor and breast cancer is exactly the same as observed in sclerosing pneumocytoma. In juvenile granulosa cell tumor, the $A K T 1$ internal tandem duplication was detected in 10 out of 16 case (63\%). In addition, there was also a high frequency of recurrent $A K T 1$ point mutations (10 out of 16 cases, 63\%) [23]. However, unlike in sclerosing pneumocytoma, the $A K T 1$ internal tandem duplication and point oncogenic transformation [22]. 
Table 3 Characteristics of patients with $A K T 1$-internal tandem duplications or other forms of $A K T 1$ mutations in the VGH-TPE cohort

\begin{tabular}{|c|c|c|c|c|}
\hline & $\begin{array}{l}\text { AKT1-internal tandem } \\
\text { duplication }(n=20)\end{array}$ & $\begin{array}{l}\text { Other } A K T 1 \text { mutations } \\
(n=20)\end{array}$ & $\begin{array}{l}\text { Total } \\
(n=40)\end{array}$ & $P$-value \\
\hline $\begin{array}{l}\text { Age (year, mean } \pm \text { standard } \\
\text { deviation) }\end{array}$ & $52.7 \pm 13.8$ & $47.7 \pm 13.5$ & $50.2 \pm 13.7$ & $0.258^{\mathrm{a}}$ \\
\hline \multicolumn{5}{|l|}{ Sex } \\
\hline Female & $20(100 \%)$ & $16(80 \%)$ & $36(90 \%)$ & $0.106^{\mathrm{b}}$ \\
\hline Male & $0(0 \%)$ & $4(20 \%)$ & $4(10 \%)$ & \\
\hline Location & & & & $0.113^{\mathrm{b}}$ \\
\hline Right lung & $13(65 \%)$ & $8(40 \%)$ & $21(53 \%)$ & \\
\hline Left lung & $7(35 \%)$ & $12(60 \%)$ & $19(48 \%)$ & \\
\hline $\begin{array}{l}\text { Tumor size }(\mathrm{cm}, \text { mean } \pm \\
\text { standard deviation })\end{array}$ & $2.0 \pm 1.2$ & $2.3 \pm 1.3$ & $2.2 \pm 1.2$ & $0.460^{\mathrm{a}}$ \\
\hline Histology pattern & & & & $0.985^{\mathrm{b}}$ \\
\hline Hemorrhagic & $6(30 \%)$ & $6(30 \%)$ & $12(30 \%)$ & \\
\hline Papillary & $7(35 \%)$ & $6(30 \%)$ & $13(33 \%)$ & \\
\hline Sclerotic & $1(5 \%)$ & $1(5 \%)$ & $2(5 \%)$ & \\
\hline Solid & $6(30 \%)$ & $7(35 \%)$ & $13(33 \%)$ & \\
\hline
\end{tabular}

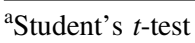

${ }^{\mathrm{b}}$ Chi-squared test or Fisher's exact test

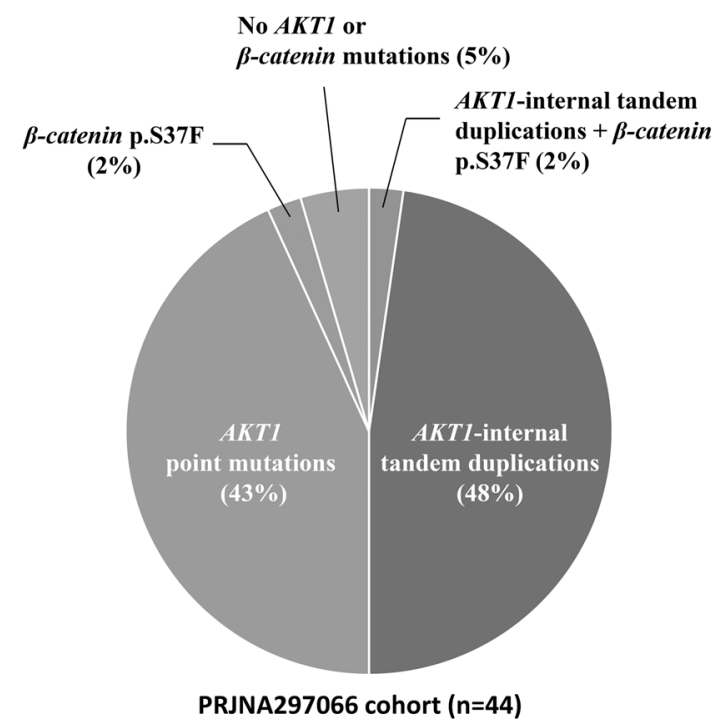

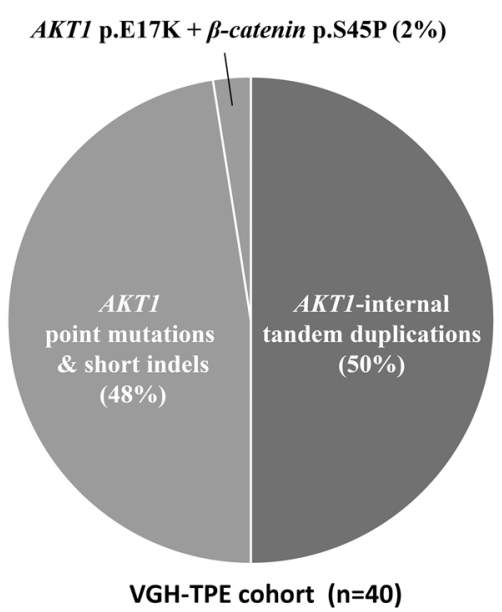

Fig. 5 Pie charts of $A K T 1$ and $\beta$-catenin mutations in the PRJNA297066 and VGH-TPE cohorts mutations in juvenile granulosa cell tumor do not appear to be mutually exclusive. Another interesting finding is that the sites of $A K T 1$ point mutations in sclerosing pneumocytoma are restricted to the Pleckstrin homology domain, whereas in juvenile granulosa cell tumor, many $A K T 1$ point mutations occur outside the Pleckstrin homology domain. In breast cancer, the existence of $A K T 1$ internal tandem duplication was identified very recently in a large-scale study by Chang et al. [24]. In this study, $A K T 1$ point mutations (p.E17K) were detected in 80 out of 2561 cases (3\%), whereas $A K T 1$ internal tandem duplications were only detected in 2 out of 2559 cases
$(0.08 \%)$, which is very intriguing. In sclerosing pneumocytoma and juvenile granulosa cell tumor, the frequencies of AKT1 internal tandem duplications and point mutations were nearly equal. However, there appears to be a substantial difference between the frequency of $A K T 1$ point mutations and $A K T 1$ internal tandem duplications in breast cancer.

The pathogenic mechanisms of AKT1 internal tandem duplications are not well understood. It is noticeable that the insertion points of duplicated sequences in AKT1 internal tandem duplications clustered at amino acids 76-85 of AKT1 protein, which contains the region of 
Fig. 6 Representative histological features of cases with $A K T 1$ point mutations or short indels (a, c, e, $\mathbf{g}$ ), and cases with $A K T 1$ internal tandem duplication $(\mathbf{b}, \mathbf{d}, \mathbf{f}, \mathbf{h})$. a A case (V01T) with $A K T 1$ p.E17K mutation showing papillary pattern. b A case (V26T) with AKT1-internal tandem duplication showing papillary pattern. c A case (V07T) with $A K T 1$ p.E17K mutation showing hemorrhagic pattern. d A case (V19T) with $A K T 1$-internal tandem duplication showing hemorrhagic pattern. e A case (V12T) with $A K T 1$ p.Q79K; p. W80R mutation showing solid pattern. f A case (V35T) with AKT1-internal tandem duplication showing solid pattern. g A case (V32T) with AKT1 p.W80R; p.

L78_Q79insKK mutation showing sclerotic pattern. h A case (V18T) with $A K T 1$-internal tandem duplication showing sclerotic pattern (original magnification: $\times 200)$
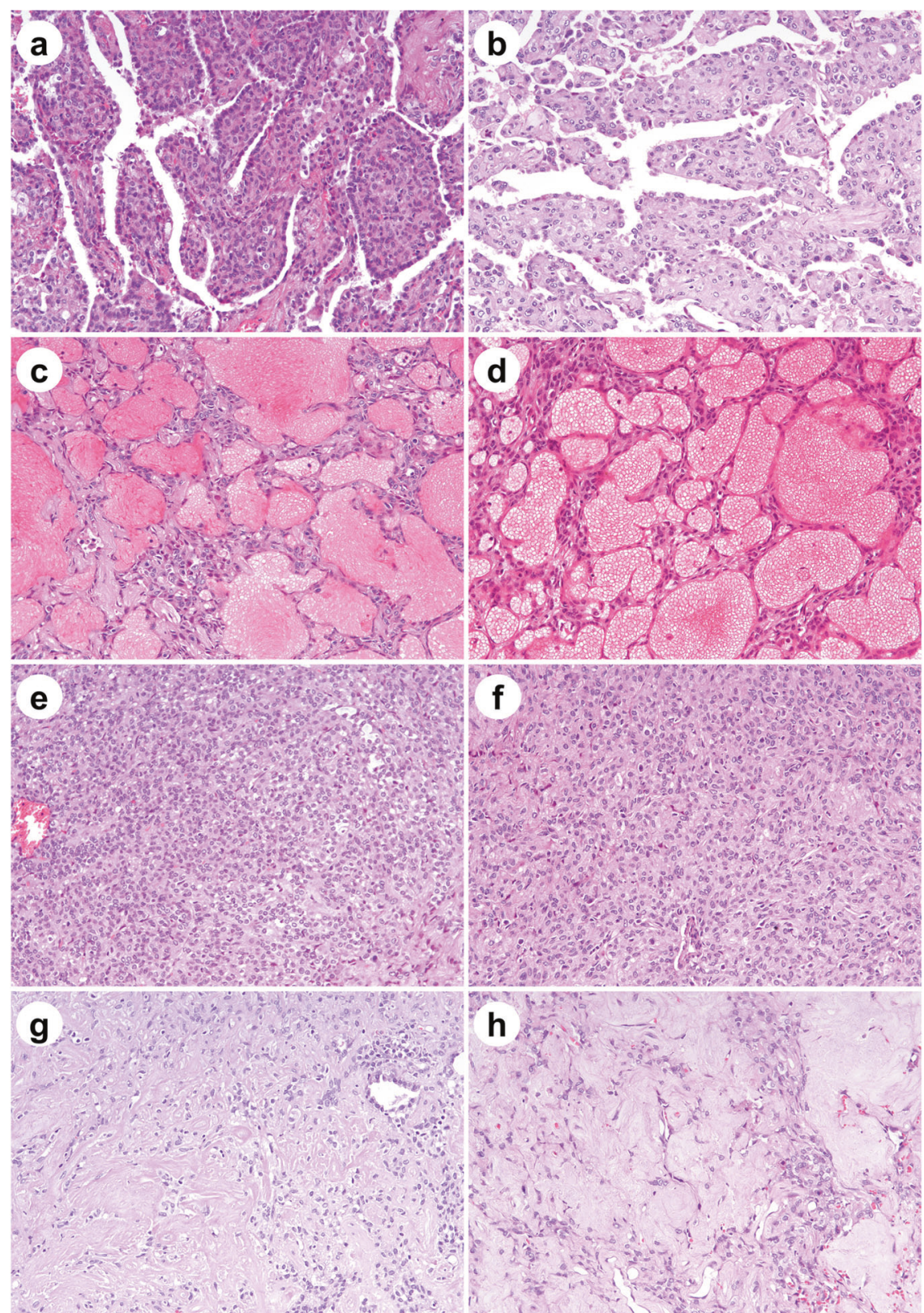

Pleckstrin homology domain and kinase domain interaction interface (Fig. 7) [19]. It has been shown that disruption of Pleckstrin homology domain and kinase domain interactions by point mutations at the Pleckstrin homology domain and kinase domain interaction interface leads to oncogenic activation of AKT in human cancers [19]. It seems plausible that the AKT1 internal tandem duplications occurring at this region might also disturb Pleckstrin homology domain and kinase domain interactions, and lead to oncogenic activation of AKT. To verify this speculation, further structural studies are required. However, in studies on cell lines, it has been shown that HeLa cells and human mammary gland cells (MCF10A) transfected with $A K T 1$ internal tandem duplication mutation constructs display high level of AKT1 phosphorylation and activation [23, 24]. Furthermore, cells transfected with AKT1 internal tandem duplication mutation constructs demonstrated sensitivity to the AKT kinase inhibitor, AZD5363, indicating the therapeutic implications of $A K T 1$ internal tandem duplication [24, 25].

In conclusion, our results show that $A K T 1$ mutations, including internal tandem duplication, point mutations, and short indels, are present in nearly all sclerosing pneumocytomas. These mutations are mutually exclusive of each other. All these mutations occur in the Pleckstrin homology domain, a critical component in the activation 


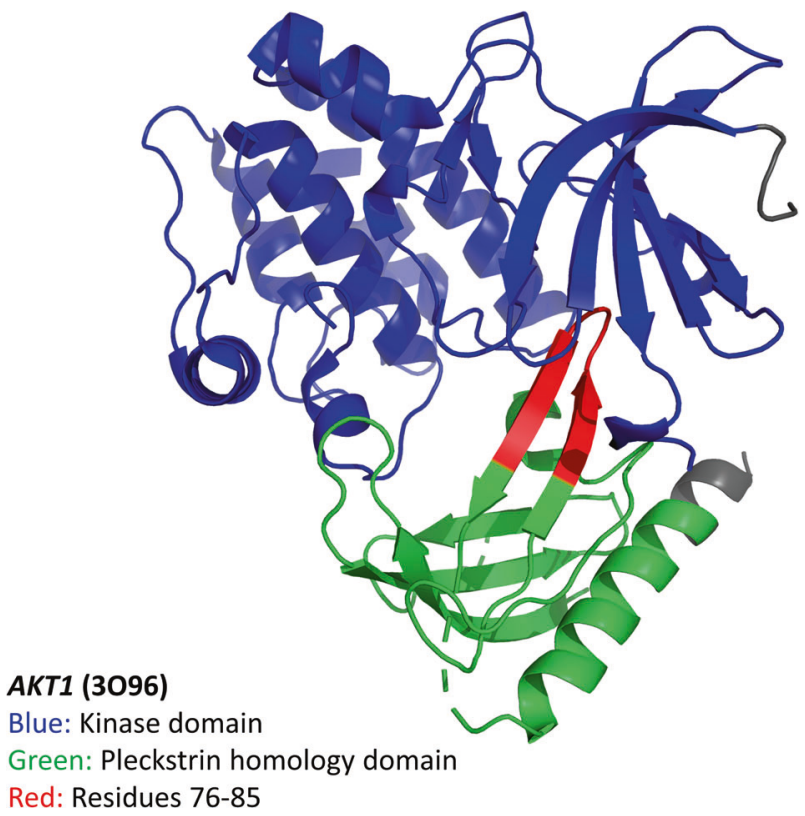

Fig. 7 Ribbon representation of Pleckstrin homology domain (green) and kinase domain (blue) of $A K T 1$ protein (Protein Data Bank ID code 3096). The insertion points of duplicated sequences in $A K T 1$ internal tandem duplication clustered at amino acids 76-85 (red), which involve the region of the interaction interface between the Pleckstrin homology domain and kinase domain

of the $A K T 1$ protein. Our results suggest that $A K T 1$ mutation is the genetic hallmark of sclerosing pneumocytoma.

Acknowledgements The authors are grateful to Wen-Yu Hsieh and Chun-An Kuo for providing technical assistance, and to the Biobank, Taipei Veterans General Hospital for assistance with sample preparation in this study. This work was supported by Ministry of Science and Technology, Taiwan (MOST 106-2221-E-010-019-MY3, MOST 1072320-B-075-004), and Taipei Veterans General Hospital, Taipei, Taiwan (V108B-001).

\section{Compliance with ethical standards}

Conflict of interest The authors declare that they have no conflict of interest.

Publisher's note Springer Nature remains neutral with regard to jurisdictional claims in published maps and institutional affiliations.

\section{References}

1. Devouassoux-Shisheboran M, Hayashi T, Linnoila RI, Koss MN, Travis WD. A clinicopathologic study of 100 cases of pulmonary sclerosing hemangioma with immunohistochemical studies: TTF1 is expressed in both round and surface cells, suggesting an origin from primitive respiratory epithelium. Am J Surg Pathol. 2000;24:906-16

2. Adachi Y, Tsuta K, Hirano R, Tanaka J, Minamino K, Shimo T, et al. Pulmonary sclerosing hemangioma with lymph node metastasis: a case report and literature review. Oncol Lett. 2014;7:997-1000

3. Kalhor N, Staerkel GA, Moran CA. So-called sclerosing hemangioma of lung: current concept. Ann Diagn Pathol. 2010;14: 60-7.

4. Wei S, Tian J, Song X, Chen Y. Recurrence of pulmonary sclerosing hemangioma. Thorac Cardiovasc Surg. 2008; $56: 120-2$.

5. Lim JH, Lee N, Choi DW, Oh HJ, Park HY, Kim KH, et al. Pulmonary sclerosing pneumocytoma mimicking lung cancer: Case report and review of the literature. Thorac Cancer. 2016;7: $508-11$.

6. Yang $\mathrm{CH}$, Lee LY. Pulmonary sclerosing pneumocytoma remains a diagnostic challenge using frozen sections: a clinicopathological analysis of 59 cases. Histopathology. 2018;72: $500-8$.

7. Niho S, Suzuki K, Yokose T, Kodama T, Nishiwaki Y, Esumi $\mathrm{H}$. Monoclonality of both pale cells and cuboidal cells of sclerosing hemangioma of the lung. Am J Pathol. 1998;152: 1065-9.

8. Wang EH, Dai SD, Qi FJ, Hong-Tao X, Wei Q. Gene expression and clonality analysis of the androgen receptor and phosphoglycerate kinase genes in polygonal cells and cuboidal cells in so-called pulmonary sclerosing hemangioma. Mod Pathol. 2007;20:1208-15.

9. Sartori G, Bettelli S, Schirosi L, Bigiani N, Malorana A, Cavazza A, et al. Microsatellite and EGFR, HER2 and K-RAS analyses in sclerosing hemangioma of the lung. Am J Surg Pathol. 2007;31: 1512-20.

10. Chiang PM, Yuan RH, Hsu HC, Mao TL, Hu RH, Lai PL, et al. Frequent nuclear expression of beta-catenin protein but rare betacatenin mutation in pulmonary sclerosing haemangioma. J Clin Pathol. 2008;61:268-71.

11. Jung SH, Kim MS, Lee SH, Park HC, Choi HJ, Maeng L, et al. Whole-exome sequencing identifies recurrent AKT1 mutations in sclerosing hemangioma of lung. Proc Natl Acad Sci USA. 2016; 113:10672-7.

12. Yohe S, Thyagarajan B. Review of clinical next-generation sequencing. Arch Pathol Lab Med. 2017;141:1544-57.

13. Spencer DH, Abel HJ, Lockwood CM, Payton JE, Szankasi P, Kelley TW, et al. Detection of FLT3 internal tandem duplication in targeted, short-read-length, next-generation sequencing data. J Mol Diagn. 2013;15:81-93.

14. Roy A, Kumar V, Zorman B, Fang E, Haines KM, Doddapaneni, et al. Recurrent internal tandem duplications of BCOR in clear cell sarcoma of the kidney. Nat Commun. 2015;6:8891.

15. Chiba K, Shiraishi Y, Nagata Y, Yoshida K, Imoto S, Ogawa S, et al. Genomon ITDetector: a tool for somatic internal tandem duplication detection from cancer genome sequencing data. Bioinformatics. 2015;31:116-8.

16. Li H, Durbin R. Fast and accurate short read alignment with Burrows-Wheeler transform. Bioinformatics. 2009;25:1754-60.

17. Thorvaldsdottir H, Robinson JT, Mesirov JP. Integrative Genomics Viewer (IGV): high-performance genomics data visualization and exploration. Brief Bioinform. 2013;14:178-92.

18. Xu C, Gu X, Padmanabhan R, Wu Z, Peng Q, DiCarlo J, et al. smCounter2: an accurate low-frequency variant caller for targeted sequencing data with unique molecular identifiers. Bioinformatics. 2019;35:1299-309.

19. Parikh C, Janakiraman V, Wu WI, Foo CK, Kljavin NM, Chaudhuri S, et al. Disruption of PH-kinase domain interactions leads to oncogenic activation of AKT in human cancers. Proc Natl Acad Sci USA. 2012;109:19368-73.

20. Manning BD, Toker A. AKT/PKB signaling: navigating the network. Cell. 2017;169:381-405. 
21. Mundi PS, Sachdev J, McCourt C, Kalinsky K. AKT in cancer: new molecular insights and advances in drug development. $\mathrm{Br} \mathrm{J}$ Clin Pharm. 2016;82:943-56.

22. Carpten JD, Faber AL, Horn C, Donoho GP, Briggs SL, Robbins $\mathrm{CM}$, et al. A transforming mutation in the pleckstrin homology domain of AKT1 in cancer. Nature. 2007;448:439-44.

23. Bessiere L, Todeschini AL, Auguste A, Sarnacki S, Flatters D, Legois B, et al. A hot-spot of in-frame duplications activates the oncoprotein $A K T 1$ in juvenile granulosa cell tumors. EBioMedicine. 2015;2:421-31.

24. Chang MT, Bhattarai TS, Schram AM, Bielski CM, Donoghue MTA, Jonsson P, et al. Accelerating discovery of functional mutant alleles in cancer. Cancer Discov. 2018;8:174-83.

25. Hyman DM, Smyth LM, Donoghue MTA, Westin SN, Bedard PL, Dean EJ, et al. AKT Inhibition in Solid Tumors With AKT1 Mutations. J Clin Oncol. 2017;35:2251-9. 